\title{
Contact des langues dans le contexte
} sociolinguistique mozambicain

Language Contacts in the Sociolinguistic Context of Mozambique

\section{César Cumbe et Afonso Muchanga}

\section{OpenEdition}

12 Journals

Édition électronique

URL : http://journals.openedition.org/etudesafricaines/111

DOI : 10.4000/etudesafricaines. 111

ISSN : $1777-5353$

Éditeur

Éditions de l'EHESS

\section{Édition imprimée}

Date de publication : 1 janvier 2001

Pagination : 595-618

ISBN : 978-2-7132-1394-6

ISSN : 0008-0055

Référence électronique

César Cumbe et Afonso Muchanga, «Contact des langues dans le contexte sociolinguistique mozambicain », Cahiers d'études africaines [En ligne], 163-164 | 2001, mis en ligne le 21 novembre 2013, consulté le 05 février 2021. URL : http://journals.openedition.org/etudesafricaines/111 ; DOI : https://doi.org/10.4000/etudesafricaines.111 


\section{César Cumbe \& Afonso Muchanga}

\section{Contact des langues dans le contexte sociolinguistique mozambicain}

\section{Les linguistes font confiance aux femmes...}

Lors de l'élaboration, au Mozambique, des livres en langues nationales, en particulier en maconde, macua et nyanja ${ }^{1}$, Tine Van Eys et Elisabeth Sequeira (1997: 26), directeur général et directrice exécutive de l'ONG « Progresso », racontent leur expérience avec les linguistes sur le terrain :

«[...] chose intéressante, dans cette expérience nous avons remarqué que quand on faisait la vérification des expressions, mots et sens dans une langue donnée, dans les zones rurales, les linguistes font plus confiance aux femmes, ils considèrent que les hommes sont plus exposés aux interférences et que la langue est plus conservée dans la manière dont les femmes la parlent. »

Voilà posé le problème des langues en contact et des incidences subjectives au Mozambique. Prenant en considération le témoignage précité, le sexe apparaît comme un facteur non négligeable qui conditionnerait les imaginaires linguistiques. À la lumière de ces derniers, les femmes campagnardes se voient attribuer le mérite de conserver la pureté des langues. Si les linguistes leur font confiance, c'est sans doute parce qu'elles seraient imperméables aux interférences, qu'elles auraient le don naturel de « résister » à la variation linguistique, à laquelle pourtant toutes les langues sont « condamnées ». L'idée que la pureté des langues mozambicaines est mieux conservée chez les femmes des zones rurales soulève une question : s'agitil d'un mythe ou d'une réalité ?

Certes, cette idée peut comporter une part de vérité et correspondre probablement à une certaine réalité (ne serait-ce que celle des linguistes en question, après tout, ce sont des scientifiques !), mais elle est loin d'être le tout de la réalité, surtout quand on sait que du point de vue sociolinguistique, il n'existe ni langues «pures » ni langues «impures». À de rares exceptions près (peuples isolés, le Mozambique excepté donc!), toutes les langues subissent l'influence d'autres langues en contact avec elles. L'emprunt

1. Langues parlées au nord du Mozambique, plus exactement dans les provinces de Cabo Delgado, Nampula et Niassa, respectivement. 
lexical en est la marque la plus spectaculaire ; ainsi, les langues mozambicaines comportent du vocabulaire d'origine portugaise, et le portugais mozambicain comporte du vocabulaire d'origine de celles-ci. Une simple « excursion», que ce soit en ville ou à la campagne, suffirait pour s'en rendre vite compte.

Bien sûr, l'interpénétration entre les langues en contact ne se limite pas au plan lexical, en syntaxe et en phonétique on peut aussi observer des influences. D'ailleurs, même les langues assez éloignées géographiquement ne font pas exception. C'est le cas des langues balkaniques (le grec moderne, le roumain et le bulgare) qui, appartenant à des branches différentes de l'arbre indo-européen, ont vu se développer des traits syntaxiques communs. On sait également que l'anglais comporte $85 \%$ de vocabulaire d'origine latine ou française. Nous pourrions multiplier les exemples, mais cela nous éloignerait de notre propos. Pour conclure sur cette entrée dans la subjectivation des langues au Mozambique, nous remarquerons que les représentations sociolinguistiques affichées dans le témoignage précité nous renvoient à l'activité épilinguistique des sujets. Dans cette optique, certaines questions s'imposent : comment les Mozambicains, locuteurs plurilingues, dans un pays sociopolitiquement monolingue (lusophone), vivent-ils leur situation linguistique ? Que pensent-ils de leurs langues ? Comment se positionnentils face à cette pluralité de langues dans leur quotidien et face à la dynamique diglossique ? C'est ce que nous proposons d'aborder dans cet article, à partir d'une enquête sociolinguistique que nous avons réalisée dans les environs de la ville de Maputo. Mais il nous semble utile de présenter avant tout la situation linguistique du Mozambique.

\section{Vue panoramique de la situation (socio)linguistique au Mozambique}

Le Mozambique est un pays d'Afrique australe, avec une superficie de $799380 \mathrm{~km}^{2}$, une population de 17 millions d'habitants, et un littoral très réputé pour sa biodiversité ${ }^{2}$. Comme tous les pays plurilingues, le Mozambique présente une situation linguistique où, d'une part, l'émergence de multiples lectes ${ }^{3}$ alimente des discussions au sein des linguistes pour les nommer, les définir, les comptabiliser, et où, d'autre part, la concurrence de ces lectes dans le quotidien entraîne une diversité de positionnements épilinguistiques de la part des usagers. Le Mozambique est l'un de ces pays d'Afrique australe où l'on ne trouve que des langues bantoues. Et pourtant,

2. Le littoral mozambicain s'intègre dans une zone internationalement élue comme un centre d'endémisme et de biodiversité d'importance mondiale. Témoignage de Mia Couto, biologiste et écrivain, dans Indico, série II, $\mathrm{n}^{\circ}$ 11, avril-juin 2000, pp. 7-10.

3. Lecte au sens où l'entend C. CANUT (2001), c'est-à-dire, terme neutre fonctionnant comme hyperonyme de langue, sociolecte, dialecte, etc. 
lorsqu'on arrive dans le pays du « Métical », on est très vite frappé par le déséquilibre réel entre la pratique linguistique, l'option politique de la pratique linguistique et l'environnement frontalier linguistique, essentiellement anglophone. Bien sûr, le Mozambique partage des langues communes avec ses voisins : Afrique du Sud (zoulou, shangane), Swaziland (siswati), Zimbabwe (shona), Malawi (yao, lomwe), Tanzanie (swahili, makonde). Pour mieux rendre compte des dynamiques linguistiques et des dynamiques d'usages au Mozambique, nous allons privilégier trois axes : un survol historique, un inventaire des langues bantoues en présence au Mozambique, et, enfin, le statut des langues après l'indépendance.

\section{La situation linguistique au Mozambique: survol historique}

Comme nous venons de le signaler, le Mozambique a la particularité de n'avoir comme langues natives que des langues bantoues. Tout a commencé avec l'arrivée des peuples bantous, au $\mathrm{VI}^{\mathrm{e}}$ siècle, en trois vagues successives. Ils ont d'abord peuplé le nord, entre le Rovuma et le Zambèze (ethnies makhwa et yao), puis le centre et le sud, entre Zambèze et Limpopo et un troisième groupe, au-delà des actuelles frontières du Mozambique, s'est établi en Afrique du Sud, au Botswana, au Lesotho et au Swaziland.

Tous ces peuples bantous parlent des langues semblables et il y a une formidable intercompréhension, même si chaque groupe ne pratique pas nécessairement la langue de l'ethnie voisine : «La notion de peuple bantou est une notion purement linguistique. Il n'y a ni race ni ethnie bantoue, mais plutôt 300 langues que le chercheur allemand Bleek a proposé en 1862 d'appeler bantoues parce que, malgré les quelques variantes dominantes, elles utilisent toutes le mot "bantou", pour dire les gens » (Jouaneau 1995 : $11)^{4}$. Signalons que cette caractérisation extrêmement réductrice est contestée par beaucoup de linguistes contemporains qui s'intéressent à ces langues.

Effectivement, le débat sur les langues mozambicaines est aujourd'hui encore très ouvert ${ }^{5}$. Remarquons qu' «aujourd'hui, on peut parler d'une inflation pour nommer les langues parlées au Mozambique, on entend plutôt: langues maternelles, langues nationales, langues africaines, langues natives, langues bantoues, langues locales, langues mozambicaines » (Lopes 1997). Il en va de même pour le portugais. On entend plutôt : «Langue

4. D. Jouaneau a occupé plusieurs postes au Quai d'Orsay et à l'étranger, notamment celui de chargé d'affaires à Harare au moment de l'indépendance du Zimbabwe. Il a été ambassadeur au Mozambique de 1990 à 1993.

5. D'après Daniel Jouaneau, la classification des langues mozambicaines a fait l'objet de plusieurs études : Cabral en 1975, Hélène Marénis en 1981, Mateus Katupha en 1989, et l'Université Eduardo Mondlane de Maputo travaille actuellement à un inventaire sytémique. D'ailleurs, c'est justement dans cette université que siège le groupe de chercheurs qui travaillent sur les langues mozambicaines, NELIMO (Nucleo de estudo de linguas moçambicanas). 
importée, portugais mozambicain, portugais au Mozambique »(ibid.). Audelà de ces hésitations terminologiques, on reconnaît la volonté de légitimer toutes les langues parlées au Mozambique. Les langues bantoues occupent, parmi les langues africaines, une place très importante aussi bien par leur véhicularité que par le nombre de locuteurs qui les parlent. En effet, sur dix langues d'Afrique noire ayant plus de trois millions de locuteurs, quatre sont bantoues : le kirwanda, le zoulou, le shona et le emakhuwa ${ }^{6}$.

À leur arrivée, les Bantous ont trouvé sur place une population d'aborigènes constituée d'une part d'éleveurs, les Hottentots (qui se sont donnés le nom de «khoi»), d'autre part des chasseurs, les Bochimans (que les Hottentots appellent «san »). L'arrivée des Bantous au Mozambique semble avoir été conditionnée par des circonstances climatiques, du moins, c'est ce que disent les historiens et les linguistes, pour qui la migration des peuples bantous s'explique par l'assèchement du Sahara, les obligeant ainsi à se regrouper le long de la bordure sud du désert, là où sont situés le Niger d'aujourd'hui, le Nigeria, le Cameroun, le Tchad, la République Centrafricaine. Les historiens ajoutent que ces peuples sont peu à peu descendus vers l'équateur, en groupes de familles marchant à l'allure de leurs troupeaux, pour s'établir dans la région correspondant aujourd'hui au Gabon, au Congo et au Zaïre.

D'après ce qui précède, on comprend bien que quand les Portugais s'installèrent au Mozambique, au début $\mathrm{du} \mathrm{XVI}^{\mathrm{e}} \operatorname{siècle}^{7}$, le pays était déjà habité depuis plus d'un millénaire par les Bantous. C'est grâce au contact avec les autres peuples, notamment avec les Portugais et les Arabes, que les langues bantoues gagnent une nouvelle dynamique et connaissent un vrai enrichissement lexical, à tel point que certains philologues, influencés par l'esprit de l'époque, revendiquent même la parenté des langues bantoues avec les langues indo-européennes (de Nogueira: 1954).

Le contact entre les langues bantoues et celles venues d'ailleurs, en particulier le portugais, semble avoir été fructueux sur le plan lexical. Cependant, sur le plan sociopolitique, ce contact instaurerait un certain déséquilibre entre les deux communautés. Ce déséquilibre est à l'origine de l'apparition d'une situation (socio)linguistique tendue dans laquelle l'évolution des langues natives est conditionnée par les stratégies adoptées par le système colonial. À ce propos, n'oublions pas que, contrairement aux Anglais $^{8}$, les Portugais au Mozambique, dès leur débarquement, considéraient les natifs comme une communauté sans identité, sans culture, sans

6. Nous ne disposons pas d'informations de tous les pays où on parle ces langues. Cela dit, nous savons que le zoulou est parlé en Afrique du Sud, au Swaziland, au Mozambique. Le shona est parlé au Zimbabwe, au Mozambique.

7. Les Portugais sont restés au Mozambique pendant cinq siècles, jusqu'au 25 juin 1975, date de l'indépendance nationale du Mozambique.

8. La stratégie coloniale des Anglais consistait à faire croire, de façon subtile, qu'ils ne se mêlaient pas à la vie des natifs. 
langue. On voit bien que sur le plan idéologique et politique, cette vision des communautés linguistiques natives n'est rien d'autre qu'une astuce pour mieux implanter l'entreprise coloniale. En d'autres termes, explique L.-J. Calvet (1974 : 10), « cette manière de nier la langue des autres peuples, cette négation, avec d'autres, constituant le fondement idéologique de la supériorité de l'Occident sur les peuples exotiques [...]» va déboucher sur « le racisme et le colonialisme glottophage »; d'où la création des conditions propices à la glottophagie partielle du portugais sur les langues natives.

En quoi consiste la glotttophagie?

En fait, si on suit le raisonnement de Calvet, la glottophagie est une sorte de relation «cannibale» où le colon venu d'Europe, non seulement «a dévoré le colonisé », mais a également «dévoré ses langues » (ibid. : 12). «Dévorer» connote bien ici la gourmandise du colon venu d'Europe pour manger à sa faim et assimiler les peuples exotiques. Mais les faits prouveront, bien entendu, qu'assimiler un peuple n'est pas une mince affaire. Avec l'arrivée des Portugais au Mozambique, s'instaure une dichotomie conflictuelle entre la langue dominante et la langue dominée. Il y a donc, d'une part, la langue portugaise, celle qu'on peut écrire, celle qui est garante de la culture et de la civilisation; et puis il y a d'autre part « les dialectes ou les jargons grâce auxquels communiquent péniblement les peuplades que les civilisateurs vont délivrer de leur sauvagerie» (ibid. : 121). Autrement dit, le colonialisme glottophage, explique Calvet, se résume en deux dogmes :

— « Les colonisés ont tout à gagner à apprendre notre langue qui les introduira à la civilisation, au monde moderne. »

— «Les langues indigènes seraient incapables de remplir cette fonction, incapables de véhiculer des notions modernes, des concepts scientifiques, incapables d'être des langues d'enseignement, de culture ou de recherche » (ibid. : 123).

Il n'est donc pas étonnant que la politique coloniale du gouvernement portugais, en collaboration avec les missions catholiques de l'Afrique et du Timor, ait toujours mis l'accent sur la langue portugaise comme le seul moyen pour instruire, civiliser, évangéliser, enfin bref, pour « portugaiser ${ }^{9}$ les natifs.

9. Néologisme de Christopher Stroud \& Antonio Tuzine (1998), traduit du portugais par nous-mêmes, dans leur ouvrage. 
Est-ce que les objectifs coloniaux ont été atteints à travers la glottophagie?

Christopher Stroud et Antonio Tuzine (1998), citant Helgesson, rappellent que le gouvernement colonial mozambicain s'est prononcé à plusieurs reprises à propos du statut et des usages des langues au Mozambique. L'attention était, bien entendu, centrée sur la langue portugaise. Par exemple, en 1907, la loi détermine que l'instruction doit se faire uniquement en portugais, et on doit utiliser exclusivement des livres approuvés officiellement. En 1926, l'implantation de l'État nouveau a publié le statut organique des missions catholiques portugaises de l'Afrique et du Timor, à travers lequel l'église catholique, au nom de l'État portugais, devait civiliser et évangéliser la population indigène. En 1941, le statut missionnaire indiquait que l'éducation des natifs serait entièrement à la charge des missions catholiques, l'État devant s'occuper seulement des politiques ou programmes éducationnels. En 1958, encore une fois, le portugais a le statut de langue d'instruction obligatoire.

Les langues mozambicaines, quant à elles, seront bannies du système scolaire par les diplômes 167 et 168 du 3 août 1929. Tout matériel écrit en langues indigènes était strictement interdit. L'usage des langues indigènes était vu comme un obstacle, comme un danger ; car, d'une part, «les langues indigènes empêcheraient le natif de quitter le monde barbare et sauvage pour accéder au monde civilisé » et, d'autre part, « elles l'empêcheraient d'aimer le Portugal $»$ (ibid.).

Tout est clair ici, le discours colonial cherche à montrer que « la glottophagie est un fait d'évidence, inéluctable, et de plus souhaité par les colonisés eux-mêmes » (Calvet 1974: 124). La "portugaisisation » est vantée comme la seule voie d'accès à la civilisation idéale. L'objectif de la péjoration des langues locales, précisons de nouveau, était de mieux asseoir la domination de la langue portugaise, et d'une façon générale, du système colonial portugais.

Alors que les décrets visant l'abolition des cultures locales, des langues locales fourmillaient sans cesse, les militants de l'église protestante menaient un combat clandestin pour lutter contre le mépris, la dictature et le « cannibalisme» linguistique portugais. C'est ce que confirment Christopher Stroud et Antonio Tuzine (1998) : «Les missions protestantes ont réussi à organiser clandestinement des programmes éducationnels en utilisant des langues africaines. C'est ainsi que grâce à la méthode de Laubach $^{10}$, ils ont

10. La méthode de Laubach, expliquent les auteurs, qui porte le nom d'un missionnaire américain des Philippines, Dr. Frank Laubach, part du principe que les lettres feraient émerger des images simples, facilement identifiables. Par exemple, à partir d'un dessin de singe (hawu en tshwa) on fera émerger la lettre « $\mathrm{h}$ ", à partir du dessin d'une main on écrirait la lettre «c», etc. Les auteurs ajoutent que cette méthode a prouvé son efficacité et son adaptabilité dans des centaines de langues. Grâce à cette méthode, témoignent les auteurs dans les séminaires inspirés par le conseil chrétien, des adaptations ont été réalisées pour les trois langues du sud du Mozambique : ronga, tsonga et tshwa. 
pu alphabétiser les natifs, surtout, mineurs et villageois, en leur propre langue dans des classes montées clandestinement. »

Avec la fin de la colonisation portugaise, toutes les valeurs coloniales se voient rejetées, à l'exception de la langue. D'ailleurs, curieusement, celleci a été souvent utilisée comme le principal outil pour «balayer » les valeurs coloniales et laver la mémoire des taches laissées par ce passé douloureux, pour accéder à l'après deuil, à la vie nouvelle et souveraine, en tant que citoyen indépendant, libre et digne. Au lendemain de l'indépendance, c'était une chose banale d'entendre des discours, surtout dans des comices, du type :

« Abaixo Xiconhoca inimigo do povo!»

(À bas Xiconhoca ${ }^{11}$ ennemi du peuple !).

« Abaixo os assimilados! »

(À bas les assimilés !).

«Abaixo o xicote !»

(À bas le xicote ${ }^{12}$ !).

«Abaixo o trabalho forçado ! »

(À bas le travail forcé !).

« Abaixo o tribalismo!»

(À bas le tribalisme !).

«Viva FRELIMO ! »

(Vive le FRELIMO ${ }^{13}$ !).

«Viva um so povo, uma so naçao do Rovuma ao Maputo ! »

(Vive un seul peuple, une seule nation du Rovuma à Maputo !).

«Viva a unidade Moçambicana! »

(Vive l'unité mozambicaine !)...

Nous reviendrons sur l'hégémonie de la langue portugaise après l'indépendance du Mozambique. Mais avant, nous aimerions faire le point sur les langues bantoues mozambicaines.

\section{La situation linguistique au Mozambique : inventaire des langues bantoues mozambicaines}

D'après la terminologie de Guthrie, cité par José Mateus Kathupa ${ }^{14}$ (1985), les langues bantoues présentes au Mozambique, ou, tout simplement, les

11. Néologisme des politiciens mozambicains à connotation péjorative pour désigner le bourgeois imbécile.

12. Xicote, c'est le nom du bâton utilisé par la police coloniale à l'époque.

13. Front national de Libération du Mozambique ; au départ donc mouvement politique de libération nationale devenu, après l'indépendance, un parti politique au pouvoir jusqu'à aujourd'hui.

14. Linguiste et actuellement ministre de la Culture, Sports et Jeunesse. Communication lors de la première rencontre avec l'Association portugaise de linguistes qui a eu lieu à Lisbonne en 1985. 
langues bantoues mozambicaines, se distribuent en quatre zones et huit groupes :

Zone G :

G40 : ki-Swahili

Zone P :

P20 : chi-Yao et chi-Makonde

P30 : e-Mákhwa (+ e-lómwe, e-Chuwabo)

Zone $\mathrm{N}$ :

N30 : chi-Nyanja

N40 : chi-Sena

Zone $\mathrm{S}$ :

S10 : chi-Shona

S50 : chi-Tsonga (+ Changana, Ronga, Tswa)

S60 : chi-Chopi

Chose curieuse et surprenante, rien n'est dit sur la signification de ces zones, rien n'est dit non plus sur le sens des chiffres qui accompagnent la lettre de chaque zone. On sait pourtant que dans chacun de ces groupes peut être intégré un ensemble de variantes d'une même langue et non pas un ensemble de langues, car il y a une intercompréhension dans les variantes en question. C'est dans ce sens que les linguistes mozambicains, dont José Mateus Kathupa, comptabilisent en tout huit langues bantoues mozambicaines tout en précisant le nombre de locuteurs pour chaque langue:

Langue e-Mákua

Langue shi-Tsonga

Langue chi-Nyanja

Langue chi-Shona
$41 \%$ (langues e-Lómwe et e-Chuwabo comprises) $19 \%$ (langue xi-Tswa comprise) $10 \%$ (langue chi-Sena comprise) $8 \%$

Il faut reconnaître qu'il n'y a pas vraiment de consensus sur le classement, la quantification et l'orthographe des langues bantoues mozambicaines. Notre propos n'étant pas de discuter cet aspect-là, nous nous contenterons de signaler quelques divergences. Par exemple, contrairement à Kathupa (1985) qui considère en tout huit langues mozambicaines, Christopher Stroud et Antonio Tuzine (1998), prenant comme point de référence l'Atlas géographique publié par le ministère de l'Éducation en 1980, dénombrent quinze langues: makua, lomwe, marende, mwani, yao, makonde, nyanja, sena, nyungwe, shona, chagana, tswa, ronga, chope et bitonga. Ces linguistes soulèvent le problème de l'harmonisation de l'orthographe pour écrire ces langues et s'expriment en ces termes: «On ne comprend pas 
pourquoi tantôt on utilise $\mathrm{U}$ tantôt on utilise $\mathrm{W}$ ou vice versa dans des mots comme Makua, Lomwe, Chuabo, Mwani, Nyungwe ou Tswa. [..] Certaines orthographes excluent le préfixe qui désigne une langue bantoue. C'est le cas de noms comme Yao (au lieu de Ciyao), Tswa (au lieu de Xitswa), ou Chope (au lieu de Cichopi). » Les deux linguistes signalent également que le rapport du premier séminaire sur l'harmonisation de l'orthographe des langues mozambicaines postule que le Shona et le Xitsonga non seulement ont trois variantes ${ }^{15}$, mais que l' " on voit également apparaître de nouvelles désignations telles que Cibalke, Ekoti ou Cisenga ».

Par ailleurs, Michel Cahen ${ }^{16}$ (1993b) comptabilise vingt-cinq langues au Mozambique : «Il y a vingt-cinq langues, mais cela ne signifie pas que les Mozambicains, là où ils habitent, aient besoin d'un véritable arsenal linguistique pour communiquer. La situation est classique de l'Afrique : on se débrouille avec deux ou trois langues, souvent proches les unes des autres. » Daniel Jouaneau (1995: 11), à son tour, se contente de dire qu'il y a une vingtaine de langues au Mozambique sans les mentionner.

Quoi qu'il en soit, ces langues jouent un rôle de langues véhiculaires et sont parlées comme langues secondes pour une communication régionale et interethnique. Contrairement à la situation linguistique fréquente dans la plupart des pays plurilingues, on assiste au Mozambique à une totale intercompréhension grâce à la proximité génétique et géographique entre les langues bantoues. Cette double proximité est renforcée par leur fonction sociale de véhicularité, qui est souvent vantée par certains intellectuels mozambicains, comme une marque d'identité culturelle. L'exemple nous vient de Luis Bernardo Honwana (1983), écrivain de l'avant-garde, exministre de la Culture et actuellement député du FRELIMO : «C'est dans les langues bantoues où résident, se préservent et se transmettent les principaux éléments constitutifs de l'identité culturelle. »Et, pourtant, même après l'indépendance, le problème linguistique, en particulier celui du statut des langues mozambicaines, se pose encore et c'est un peu comme si le Mozambique se trouvait encore et toujours avant l'indépendance.

\section{La situation linguistique au Mozambique : le statut et les fonctions des langues après l'indépendance}

Le maintien de la langue portugaise qui, avant l'indépendance, était déjà la langue dominante, la langue d'assimilation, montre que sur le plan linguistique, au Mozambique, «après l'indépendance, c'est toujours avant l'indépendance » (Calvet $1974: 138$ ). Il est frappant de constater que le

15. Le shona a comme variantes le cimanyika, cindau et citeve et le xitsonga a comme variantes le xichangana, xironga et xitswa.

16. CNRS-Centre d'étude d'Afrique noire, Institut d'études politiques de Bordeaux, $1^{\text {er }}$ novembre 1993. 
problème des langues mozambicaines est singulièrement absent dans la pensée anticoloniale de la classe politique mozambicaine. C'est en portugais qu'a été rédigée la constitution, les lois. C'est en portugais que sont rédigés les journaux. C'est en portugais que s'annonce l'offre d'emploi et se fait l'entretien d'embauche. C'est en portugais que la police demande les papiers. C'est en portugais qu'on rend la justice. C'est en portugais que les gouverneurs prennent la parole à la radio, curieux moyen de se faire comprendre auprès du peuple majoritairement «bantouphone ». C'est en portugais que se font les campagnes d'information et de prévention par les personnels du ministère de la Santé ou de la Croix-Rouge. C'est en portugais que se font les discours politiques, les orateurs ne faisant concession aux « langues du coin » que lorsqu'il s'agit de campagnes électorales.

Le portugais, a priori censé minimiser les problèmes ethniques, perpétue d'une certaine manière l'exclusion linguistique, puisqu'il reste toujours l'apanage d'une petite élite, une langue exclusive, la langue dominante. D'une façon générale, le problème linguistique n'est posé ouvertement au Mozambique que très récemment et le plus souvent par des linguistes. Christopher Stroud et Perpétua Gonçalves (1998), par exemple, font appel à Carol Myers-Scotton pour signaler que «l'usage des langues métropolitaines par une petite élite est à l'origine du blocage pour le développement social, économique et politique de la plupart de la population ». Ils pointent des exemples concrets. C'est le cas des résultats catastrophiques, en GuinéeBissau, dus à l'usage du portugais comme langue de l'enseignement. Il en va de même du français pour l'ex-Zaïre et de l'anglais pour la Zambie et le Nigeria. Préoccupés par le vide en matière de politique et planification linguistiques adaptées à la réalité du pays, ces linguistes n'hésitent pas à faire valoir leurs conseils : «Il faut créer des conditions pour un usage plus élargi du portugais, mais aussi pour un usage de langues africaines en contextes institutionnels tels que l'éducation formelle, les tribunaux de l'État, l'administration ou les média. »

Le linguiste mozambicain, Armando Jorge Lopes (1997), lors du congrès mondial de linguistique africaine qui a eu lieu à Kwaluseni en 1994, a vivement indiqué que la politique linguistique en cours n'est plus adaptée au Mozambique de l'après-guerre ${ }^{17}$. Ceci explique pourquoi, aux yeux de

17. L'auteur parle sans doute de l'après-guerre civile. Notons que juste après l'indépendance, le Mozambique a été victime d'une très longue guerre civile pendant 25 ans. Par conséquent, l'exode rural a atteint des proportions records, à tel point que, métaphoriquement parlant, les centres urbains, souvent décrits comme les seules îles lusophones en plein océan bantouphone, ont connu une érosion considérable. Dorénavant, les langues bantoues résonneront fort autant en ville qu'à la campagne, et le portugais, suite à ces mutations sociales, prendra un nouveau destin, il se naturalisera, il sera parlé « à la mozambicaine », d'où les appellations comme le portugais mozambicain, le portugais du Mozambique, le portugais au Mozambique. Ce n'est qu'à partir du 4 septembre 1992, avec la signature de l'accord de cessez-le-feu entre les deux partis en conflit, le FRELIMO et le RENAMO, à Roma, que le Mozambique a connu la paix. 
ce linguiste, il faut promouvoir les droits des langues indigènes, développer les variétés non natives des langues (le portugais mozambicain!), des méthodes d'enseignement des langues autochtones, des politiques curriculaires ou l'alphabétisation; il faut surtout, explique-t-il, définir réellement la politique et planification linguistiques adaptées. Soucieux de l'avenir linguistique du Mozambique et tout à fait conscient que l'actuelle situation linguistique résulte des accidents politiques, économiques et historiques, Lopes ajoute que l'hégémonie linguistique du portugais, au détriment des autres langues parlées au Mozambique, justifie le complexe linguistique qui fait que tout le monde prétend avoir le portugais comme langue maternelle.

Tous ces propos convergent en un point : les intellectuels mozambicains, en particulier les linguistes, tout en reconnaissant le rôle capital du portugais, critiquent son emprise par rapport aux langues bantoues, parlées par l'écrasante majorité de la population.

Au lendemain de l'indépendance, le gouvernement mozambicain mettra l'accent plutôt sur la libération du contrôle économique ${ }^{18}$ et sur l'émancipation sociale ${ }^{19}$. Nos propos trouvent leur argument du fait que, avec l'indépendance du Mozambique, le 25 juin 1975, beaucoup de choses ont changé sur le plan social, économique, politique... Mais, sur le plan (socio)linguistique, il n'y a pas eu un véritable renversement de situation. Comme dans la plupart des pays d'Afrique nouvellement indépendants, le gouvernement s'est contenté de la langue des colons comme langue d'unité nationale, langue de communication officielle, langue d'enseignement. Il faut signaler que ce choix pragmatique était une manière de surmonter les contradictions d'ordres régional et politique auxquels le FRELIMO était confronté pendant la lutte de libération nationale; c'est ainsi que la langue portugaise gagne une dimension historique et un rôle politique de langue unificatrice du peuple Mozambicain. Après l'indépendance, on comprend bien que la déclaration politique sur le statut et les fonctions de la langue portugaise repose sur une politique linguistique implicite, dans le sens où il n'y a pas un seul document officiel où l'État déclare clairement le statut et le rôle des langues bantoues mozambicaines.

Effectivement, le choix de la langue portugaise est une sorte de dépannage pour faire face à la réalité linguistique du pays qui, sociolinguistiquement, peut être caractérisé comme un pays plurilingue. Le cas de figure de ce plurilinguisme est résumé par José Mateus Kathupa (1985) en ces termes : «L'usage de la langue portugaise $(24,4 \%$ de la population, dont $1,2 \%$ a le portugais comme langue maternelle ; $23,2 \%$ parlent le portugais

18. C'est ce dont témoigne la politique de nationalisations, pendant le gouvernement de transition en 1974, dirigé par Joaquim Alberto Chissano, qui est actuellement le président de la République et de l'OUA (Organisation de unité africaine).

19. Création de l'OMM (Organisation de la femme mozambicaine; aujourd'hui, il y a même un ministère de la Femme mozambicaine) ; création de l'OJM (Organisation de la jeunesse mozambicaine) ; création des groupes «dynamisateurs » dans les quartiers et dans les villages; création de coopératives de consommation, etc. 
et une langue bantoue). L'usage des langues bantoues $(75,6 \%$ de la population parlent uniquement en langue(s) bantoue(s)). »

Notons ici que Kathupa se réfère au recensement de 1980. Cependant, dans un article très récent, Perpétua Gonçalves (1993), linguiste, universitaire et chercheur travaillant sur les particularités du portugais parlé au Mozambique, explique que le Mozambique compte seize millions d'habitants, dont $95 \%$ ont une langue de la famille bantoue comme langue maternelle. Elle ajoute que parler portugais, aujourd'hui, au Mozambique, signifie donc qu'on appartient à une minorité qui a eu accès à l'école $(25 \%)$ et qui habite dans les centres urbains.

Paradoxalement, bien que $25 \%$ seulement de Mozambicains parlent la langue portugaise, cela n'empêche pas pour autant qu'elle soit la seule langue de l'État, de l'enseignement de l'alphabétisation, des médias, en particulier de la presse écrite. Virgilio de Lemos (1993), écrivain mozambicain réfugié en France depuis les années 1960, pour des raisons politiques, et travaillant actuellement à Radio France internationale, va plus loin en signalant qu'avec la récente adhésion du Mozambique aux pays du commonwealth, la langue portugaise au Mozambique est de moins en moins enseignée, laissant la place à l'anglais, qui devient de plus en plus important dans les régions d'Afrique australe.

Avant le déclenchement de la guerre civile et (aujourd'hui hélas !) les catastrophes naturelles, la situation linguistique mozambicaine était facile à décrire sur le plan géographique. Effectivement, la langue portugaise était plus parlée en ville qu'à la campagne, tandis que les langues bantoues étaient plus parlées à la campagne qu'en ville. À l'heure actuelle, puisque un bon nombre de «bantouphones» se trouvent en ville, surtout dans les quartiers périphériques, on assiste au Mozambique à de nouvelles dynamiques linguistiques et dynamiques d'usages.

Tout compte fait, on voit que, après l'indépendance, l'État non seulement donne un statut valorisant à la langue portugaise, mais il lui attribue également des fonctions de prestige. Paradoxalement, rien n'est dit sur le statut et les fonctions des langues natives, désormais baptisées dialectes, landim $^{20}$ souvent par influence directe de l'État même, car ces langues seront officiellement interdites (jusqu'à aujourd'hui) à l'école, au travail. Elles seront plus ou moins exclues également, cette fois-ci non pas de façon officielle, mais plutôt de façon officieuse, par une sorte d'auto-dénigrement, à tel point que certaines personnes complexées ont honte d'avouer que le portugais n'est pas leur langue maternelle. Même si ce n'est pas une tendance générale, l'auto-dévalorisation des langues bantoues fait que certaines personnes éprouvent un malaise à parler leur langue maternelle dans les espaces publics (bus, marchés, magasins...) et, parfois même dans les espaces

20. Terme générique, à l'origine utilisé par les Portugais pour désigner les parlers des indigènes (les langues bantoues mozambicaines). C'est un peu l'équivalent du patois. 
privés, intimes comme, par exemple, dans la famille, où des parents complexés, surtout dans les villes, interdisent catégoriquement à leurs enfants de parler les langues bantoues.

Ces prohibitions prouvent que ces langues, dans l'imaginaire linguistique des parents en question, jouissent d'une réputation négative et sont péjorées. Elles sont souvent taxées de «langues d'inculture », «langues vulgaires », et même « langues de chien ». À noter qu'au départ, ces valeurs étaient véhiculées par les Portugais. En effet, la terminologie coloniale pour désigner les parlers des autochtones tournait autour d'appellations comme dialectes, langues indigènes, langues natives, langues autochtones, langues des Noirs, avec une connotation très négative. À l'école, c'était essentiellement le terme landim. Curieusement, à l'exception de landim, les Portugais utilisaient (naïvement ?) des termes scientifiques (linguistiques) pour nommer les parlers qu'ils considéraient inférieurs et sans reconnaissance. Les valeurs négatives associées aux langues bantoues semblent revenir chez certains parents par effet de feed-back. Même si toutes ces appellations péjoratives réactualisées (inconsciemment) visent à décourager l'apprentissage des langues bantoues par les enfants, il ne serait pas étonnant que ces mêmes enfants, une fois grands, contredisent leurs parents, lors d'une prise de conscience linguistique. D'ailleurs, plus loin, nous verrons un exemple qui illustre nos propos. Pour l'instant, nous aimerions revenir un petit peu sur cette relation tendue entre le portugais et les langues natives. Au fond, sociolinguistiquement parlant, nous avons affaire à une diglossie, c'est-à-dire à la cohabitation plus ou moins conflictuelle entre les langues ou variétés de langues réparties socialement de façon inégalitaire. On trouve une langue ou variété de langue valorisée au détriment d'une autre.

Dans cette optique, le portugais est la langue dominante sociopolitiquement, puisque c'est la langue de l'État, de l'école, des médias, du pouvoir, de la promotion sociale... Mais statistiquement, c'est une langue très minoritaire parlée essentiellement par la population scolarisée et urbaine. En revanche, les langues bantoues, sur le plan statistique, sont dominantes, alors que sur le plan sociopolitique elles sont dominées. On comprend pourquoi affirmer que le Mozambique est un pays lusophone fait sourire beaucoup d'intellectuels avertis, en particulier des linguistes et des sociolinguistes. Pour ne prendre qu'un exemple, Armando Jorge Lopes (1997: 43) trouve qu'il est « excessif et grossier d'affirmer que le Mozambique est un pays lusophone ». Effectivement, compte tenu du nombre de locuteurs qui parlent portugais, le Mozambique est loin d'être un pays lusophone, c'est plutôt un pays bantouphone. Il serait naï de ne pas reconnaître que cette appellation pose un autre problème, car non seulement on n'est pas d'accord sur le nombre de langues bantoues parlées au Mozambique, mais on ne sait pas très bien non plus combien de locuteurs parlent telle ou telle langue bantoue. Ces discussions dépassant largement le cadre de notre article, nous nous contenterons de reconnaître que, même si les données statistiques sur le nombre de lusophones ne sont pas homogènes, une chose est certaine : le 
portugais connaît d'importantes limites géographiques (il y a peu de pays réellement lusophones, au sens sociolinguistique du terme) et sociales (le portugais est l'apanage d'une élite intellectuelle et urbaine).

$\mathrm{Si}$, au Mozambique, la langue portugaise a un certain prestige, pour ne pas dire un prestige certain, et des fonctions bien précises, c'est pour des raisons historiques et sociologiques qui tiennent à la forme du pouvoir, à l'organisation de la société. La politique linguistique coloniale trouve son écho dans la politique linguistique adoptée par le gouvernement mozambicain, qu'à la veille de l'indépendance, le plurilinguisme s'efforcera d'écarter au nom d'une unité linguistique et sociale. Le rapport des langues, dans les situations plurilingues ou multilingues, est souvent décrit en termes de conflits, en termes de guerre entre les langues. Par exemple, L.-J. Calvet (1987 : 281) va jusqu'à affirmer que « le monde, plurilingue dès ses origines est donc de par ce plurilinguisme même le lieu d'un vaste conflit sémiotique, d'une tension permanente entre le grégaire et le véhiculaire, la langue de la maison et celle du pain, les langues de pouvoir et celles de minorité ». Cette affirmation fait appel à des réserves, car si la pluralité linguistique suscite des conflits sur le plan sociopolitique, cette même pluralité est aussi perçue par certains sujets confrontés à cette réalité comme une richesse, comme un atout indispensable, c'est ce que nous verrons plus loin. La guerre des langues n'est que le reflet des guerres politiques, idéologiques ou religieuses.

Si certaines personnes prétendent que le portugais est leur langue maternelle, si certains parents refusent d'apprendre à leurs enfants une langue bantoue, c'est parce que «l'histoire linguistique de la famille est le produit de l'histoire sociale»(ibid.). Tout cela est donc «le produit du racisme ambiant, d'une dévalorisation idéologique de leur langue » (ibid.), qui dure depuis la colonisation portugaise, et les gens en question prétendent mettre fin à cette guerre linguistique par abandon des langues natives. Il s'agit, bien entendu, d'une solution non généralisée par laquelle les langues mozambicaines peuvent difficilement disparaître. Au contraire, elles semblent revenir avec force, puisqu'on leur réclame une légitimation, une valorisation. De façon modeste et à titre expérimental, elles sont déjà partie prenante, à Gaza et à Tete, depuis 1995, dans 5 écoles primaires.

\section{L'enquête sociolinguistique : approche du terrain par entretien semi-directif}

\section{Objectifs}

Si les études des dynamiques d'usages dans les situations de plurilinguisme ne sont pas très nombreuses en général, elles sont quasi inexistantes au Mozambique. C'est pourquoi nous considérons cette entreprise comme un défi que nous comptons relever. Dans le cas spécifique de cette réflexion, 
nous entendons illustrer notre propos par l'analyse d'une « micro-situation » de contact de langues dans le contexte sociolinguistique mozambicain, celle de Boane, un petit village proche de Maputo, où nous avons mené une enquête dont l'objectif était justement de voir comment, à travers l'action des langues en contact, s'illustre le positionnement des sujets enquêtés, notamment par leurs représentations, et comment ils mettent en place un certain discours sur les langues en présence, sur eux-mêmes et sur leur rapport à ces langues.

\section{Cadre de l'entretien}

L'enquête a été menée le 19 avril 2000, en portugais, à l'école secondaire Joaquim Chissano à Boane, une bourgade située à une trentaine de kilomètres de la capitale du Mozambique, Maputo. Le procédé de collecte de données utilisé est celui d'entretiens semi-directifs; nous avons en tout interviewé huit sujets, quatre filles et quatre garçons, du même niveau scolaire (seconde), dont l'âge va de 17 à 19 ans. Les entretiens ont eu lieu à la cantine de l'école, dans un climat assez détendu, même si le cadre scolaire était d'une certaine manière toujours omniprésent. Les questions tournaient autour de quelques axes prédéterminés, à savoir l'origine géographique du sujet et des parents, le capital linguistique du sujet et de ses parents, sans oublier bien sûr la répartition fonctionnelle de ce capital (quand parler telle ou telle langue ? où ? avec qui ?).

Nos questions avaient le souci de rechercher systématiquement les représentations sociolinguistiques, les positionnements subjectifs des sujets face aux différentes langues auxquelles ils sont confrontés. Le choix du public n'a pas été fait au hasard; nous voulions un public qui soit au «carrefour » des langues, un public qui soit au cœur d'un contexte de rencontre de langues. Et Boane offre ces conditions, car c'est un village «mi-urbain» et «mi-rural». Effectivement, sa relative proximité avec Maputo, à une trentaine de kilomètres, et le fait qu'il se situe sur le corridor MaputoJohannesburg, fait qu'il subit une influence constante de la capitale. En même temps, c'est la campagne profonde, et on y pratique essentiellement l'agriculture. Dans ces conditions, les élèves de l'école secondaire de Boane constituent une cible pertinente pour étudier la problématique du contact des langues.

Analyse sociolinguistique

Peut-on parler d'une conscience linguistique de la problématique du contact des langues? 
Comme nous l'avons déjà signalé, pendant et après la colonisation portugaise il y a toujours eu une survalorisation de la langue portugaise au détriment des langues bantoues mozambicaines, populairement appelées dialectes ou landim et, par conséquent, condamnées au mépris. Cependant, à croire certains propos tenus par nos enquêtés, on peut admettre, sous réserve, bien entendu, qu'il y a un certain fléchissement de tendance. En effet, nous avons été surpris par une certaine prise de conscience qui se traduit par des représentations positives à l'égard de langues bantoues, et on peut se demander si les parents de nos informateurs ont les représentations sociolinguistiques de ces langues. Les enquêtes que nous envisageons de réaliser auprès des parents des élèves devraient nous apporter des renseignements. Pour l'instant, nous ne pouvons que soulever quelques questions : comment les parents perçoivent-ils les langues bantoues ? Ont-ils des complexes à les apprendre à leurs enfants ? Partagent-ils les mêmes points de vue que nos informateurs? Ne tiennent-ils pas des discours contradictoires ? Autant de questions pour lesquelles nous espérons obtenir des éléments de réponses sur le terrain ${ }^{21}$.

Pour revenir à nos informateurs, chose intéressante, nous avons constaté que leurs représentations positives sur les langues bantoues n'impliquent pas nécessairement un positionnement de contrepoids ou de «réaction contre » la langue officielle, la langue venue d'Europe. Cette représentation positive est également assumée, non sans acuité, par les élèves auxquels les parents interdisent de parler landim, alors que, eux-mêmes, parlent ce landim, ce qui peut paraître paradoxal. Nos enquêtés n'hésitent pas à reprocher, culpabiliser et responsabiliser leurs parents. Nous citerons trois exemples :

\section{Exemple 1}

L'enquêté «B », 19 ans, déclare parler portugais, changana, et comprendre un peu le bitonga ${ }^{22}$, langue de ses parents :

L'enquêteur ${ }^{23}$ : Quelle est la langue de ta préférence parmi/ donc entre le portugais, le manhembane comme tu dis et le changana?

L'enquêté : J'aimerais parler les trois, étant donné que...

L'enquêteur: Oui, oui, je vois.

L'enquêté : Mais la langue que j'aimerais vraiment parler c'est celle que mes parents parlent, le manhembane.

21. Après avoir fait une enquête auprès des élèves de «Boane", nous envisageons d'en réaliser une autre auprès de parents, cette fois-ci en langues locales (essentiellement, « ronga » « changana » et «chope », langues que nous maîtrisons également), puisque, d'après nos témoins, la plupart des parents ne maîtrisent pas le portugais.

22. Qu'on appelle aussi «manhembane », un terme qui a une charge péjorative associée à l'avarice.

23. Nous-mêmes. 


\section{Que retenir de ce petit extrait?}

Le message est clair. Sans que la langue portugaise soit rejetée, nous assistons à une valorisation de la langue des parents, qui prend même un goût de réappropriation, ne serait-ce que sur le plan symbolico-affectif, qui ne coïncide pas vraiment avec les attitudes; notons que dans son discours, notre informateur met sur le même plan les langues auxquelles il est confronté d'une façon ou d'une autre: "J'aimerais les parler toutes les trois »; notre interlocuteur, qui déclare volontiers se sentir plus à l'aise quand il communique en portugais, se reprend comme si, par mégarde, il avait enfreint une règle de conduite familiale, symbolique et sociale qui ne peut faire table rase de la langue et de l'origine de ses parents : "Mais la langue que j'aimerais vraiment parler, c'est celle de mes parents, le manhembana. »

\section{Exemple 2}

L'enquêtée «C », 18 ans, déclare parler trois langues: le changana ${ }^{24}$, le portugais et le chope ${ }^{25}$. Sa préférence va pour le changana (ou ronga dans ce cas précis) :

L'enquêtée: Ah ! Ma langue/ de préférence j'aime parler le ronga, parce qu'ici beaucoup de gens parlent ronga.

L'enquêteur : Ouais...

L'enquêtée : Yah ! J'aime [...] ronga/ c'est vrai que j'ai quelques difficultés// Yah !/ le portugais aussi// tout n'est-ce pas...

Au premier abord la langue «élue » par l'enquêtée est le ronga/changana. Ici, il est sans doute intéressant de remarquer que les raisons de l'attachement (symbolico-affectif) à une langue donnée sont d'un autre ordre que celles qui semblent orienter l'enquêté « B »; tandis que celui-ci met l'accent sur l'attachement familial («la langue de mes parents»), cette dernière met l'accent sur la socialisation à travers la langue : «Ici beaucoup de gens parlent ronga. »

Comme par un effet de miroir, l'enquêtée « $\mathrm{C}$ » suit une ligne de pensée réciproque à celle de l'enquêté « $\mathrm{B} »$, ce qui, tout compte fait, donne le même résultat; elle commence par déclarer la langue de sa préférence (le ronga) ; mais, comme si cette déclaration limitait l'enquêté « $\mathrm{B}$ » dans sa compétence de sujet portant des identités plurielles, elle rectifie le tir, et on ne peut rester indifférent à cet effet de gradation : «J'aime parler [...] ronga/ c'est vrai que j'ai quelques difficultés// Yah !/ le portugais aussi// tout n'est-ce pas...»

24. Tantôt elle l'appelle changana, tantôt ronga, étant donné la très grande ressemblance entre ces deux langues, par leur proximité génétique et géographique, comme nous l'avons déjà vu.

25. La langue de ses parents. 


\section{Exemple 3}

L'enquêtée «A », 19 ans, dit parler portugais et sena ${ }^{26}$. Ces deux langues ont des fonctions spécifiques et la deuxième est celle qu'elle considère comme «sa » langue, le portugais, langue officielle, étant relégué au statut d'une «langue trouvée comme ça». Elle n'hésite donc pas à valoriser sa langue par opposition au portugais :

L'enquêteur : Eh/ qu'est-ce que tu penses de ceux qui disent/ ah nos langues sont des dialectes//.

L'enquêtée: Je pense qu'ils/ n'ont pas encore compris ce que signifie une langue nationale. Notre dialecte/ je ne sais pas pour les autres personnes mais pour moi, je considère plus ma langue/ eh mon dialecte que les autres langues, que le portugais, parce que le portugais c'est une langue trouvée comme ça pendant// eh (... ?)/ oui, c'est la langue officielle mais...

Un fait qui a attiré notre attention dans cet extrait, c'est la réaction de notre informatrice contre le fait qu'on appelle les langues bantoues mozambicaines «dialectes » (en réponse bien sûr à la question posée). La représentation positive sur «sa » langue, le sena, est bien évidente, mais elle est bousculée par une formulation bien ancrée dans l'esprit de l'enquêtée, à savoir la tendance à appeler «dialecte » toute langue non européenne; à noter par exemple, la reformulation de cette phrase : «Je considère plus ma langue/ et mon dialecte que les autres langues. » S'agirait-il d'une valorisation folklorisante de la langue dominée qui ne fait que conforter la position de la langue dominante?

En tout cas, on voit bien le conflit entre le $\operatorname{perçu}^{27}$ et le souhaitée ${ }^{28}$.

\section{Alternance linguistique et regards subjectifs}

Il y a une question que l'on n'a pas manqué de poser à nos interlocuteurs et qui interpelle les sujets sur la façon dont ils vivent le fait d'être confrontés à plusieurs langues dans leur vie quotidienne. De prime abord, on s'attendait à ce que les enquêtés manifestent massivement une attitude reflétant un conflit entre les langues, qui résulterait d'une tension intérieure soit en faveur du portugais, soit en faveur de la langue bantoue parlée par chaque locuteur. En réalité, les réponses que nous avons obtenues nous indiquent qu'il ne semble pas possible de lire chez les enquêtés une dynamique conflictuelle latente, à l'exception toutefois de l'enquêtée « $\mathrm{A} »$. Pour être

26. Langue de la province centrale de Sofala, dont la capitale est Beira.

27. La langue sena est perçue par notre interlocuteur comme un dialecte, un patrimoine dévalorisé, sous-estimé, en déclin, ce qui suscite une insatisfaction.

28. Le souhait de notre interlocuteur, c'est que le sena soit une langue valorisée, estimée. 
plus précis, nous citerons quelques exemples significatifs sur la gestion de la pluralité linguistique, apparemment bien vécue :

L'enquêté «B » : Je trouve que c'est un exercice simple (le fait de «jongler» avec le portugais et le chagana, dans ma vie quotidienne).

L'enquêté «D»: Ne parler qu'une seule langue ce n'est pas bien.

L'enquêteur: Pourquoi ce n'est pas bien ?

L'enquêté « $\mathrm{D} »$ : Ce n'est pas bien, parce qu'une personne peut naître ici (à Boane)/ et pas mourir ici (à Boane)/ elle peut voyager un jour.

L'enquêté « $\mathrm{G} »$ : Le ronga aussi doit être utilisé comme le portugais.

L'enquêteur : Pourquoi?

L'enquêté « $\mathrm{G} »$ : Ce sont toutes des langues, les deux sont utiles.

Remarquons que ces extraits montrent bien que les sujets sont conscients de l'alternance linguistique, dans la mesure où ils déclarent avoir une langue de préférence comme nous l'avons vu plus haut; ils semblent bien vivre le contact des langues auxquelles ils sont confrontés. S'il faut admettre que le contact des langues implique nécessairement un certain regard à l'égard de ces mêmes langues, un certain positionnement et des représentations qui n'émergent pas à la surface lors d'un simple entretien, nous croyons pouvoir conclure que les sujets arrivent à gérer leur situation et y trouver leur compte dans cet univers fort complexe. C'est en effet avec désinvolture que l'enquêté « $\mathrm{B}$ » assure que «c'est un exercice simple».

Dans un pays comme le Mozambique, «condamné » à l'ouverture, où la nécessité de la « rencontre de l'autre » semble plus que jamais d'actualité, les jeunes se montrent sensibles à cette «ouverture »; ils constituent sans doute le groupe d'âge le plus apte à accepter d'élargir ses horizons d'aller vers «l'autre », d'où la réplique « ne parler qu' une langue ce n'est pas bien, parce qu'une personne peut naître ici et ne pas mourir ici/ elle peut voyager un jour».

La langue, on le sait, est un terrain indiscutablement fertile pour l'émergence de la subjectivité. C'est un lieu de prise de position caractérisé ici par un rééquilibrage de forces entre une langue dominante (le portugais) et une langue dominée (les langues bantoues); le sujet opère ce rééquilibrage symbolique : «Le ronga doit aussi être utilisé comme le portugais » et il va plus loin pour nous rappeler qu'une langue est aussi un instrument de communication qui s'inscrit dans une dimension utilitaire : «Les deux sont utiles. » Les fonctionnements sociolinguistiques, notamment les phénomènes de positionnements et de représentations, ne sont pas linéaires et tout le monde ne prend pas naïvement la même direction. C'est ainsi que l'enquêtée « $\mathrm{A}$ » se démarque assez nettement du positionnement des autres : «Je suis contente de parler les deux langues (le portugais et le sena), parce que même si je n'étais pas contente, je ne pourrais jamais arrêter de parler une langue. »

Ces propos traduisent une certaine attitude de résignation de l'enquêtée qui n'est pas en discordance avec les propos qu'elle-même a tenus : « [...] 
Moi je considère plus ma langue/ eh mon dialecte que les autres langues, que le portugais, parce que le portugais, c'est une langue trouvée comme ça (...).»

\section{Positionnement épilinguistique}

Les propos de certains interlocuteurs nous permettent de voir que la langue, plus qu'un simple « outil de communication », peut fonctionner comme une source de «tension épilinguistique » (Canut 1998, 2000), susceptible de déclencher une ambivalence de positionnement épilinguistique envers autrui. Effectivement, l'enquêté « $\mathrm{G}$ » dit que la langue utilisée chez lui est le portugais, contrairement à ses voisins qui parlent « ronga ». Il nous dit comment ceux-ci réagissent : «... Au début il y avait/ ils se comportaient d'une drôle de façon, parce qu'ils disaient que/ le portugais c'est/ c'est une langue pour les Blancs, et nous comme Noirs nous devrions parler toujours/ ronga. »

Ici, l'attitude des voisins de notre interlocuteur semble afficher un double positionnement épilinguistique. D'une part, ils affichent un positionnement politique, dans ce cas il s'agirait de prescrire la langue portugaise, qui se voit sanctionnée en tant que langue de pouvoir. À leurs yeux, l'emploie du « ronga » se justifierait du fait qu'il est le lecte commun, du milieu, adapté à la communication locale ; d'autre part, ils affichent un positionnement individuel/subjectif, dans ce cas, c'est l'idéal de langue et le mythe de langue « originelle » qui ressortent. Leur attitude hostile serait une sorte de réparation pour que le « ronga », leur langue du cœur, «d'ancrage spatiotemporel », ne soit pas «langue de l'oubli », «langue de l'unité perdue ». La nécessité de cette mise en abyme montre à quel point la langue est marquée à la fois par le manque de cette homogénéité initiale, et à la fois à quel point l'idiolecte est marqué par l'autre (Canut 2000).

Un autre témoignage va dans le même sens. C'est celui de l'enquêtée «F $\mathrm{F}$ qui, ne parlant pas « ronga », est mise à l'écart par ses voisins : «Mes voisins ont souvent un comportement étrange/ parfois en discutant avec eux/ sans préavis ils se mettent à parler ronga (tout en sachant que nous parlons portugais uniquement)/ [...] ils font ça, parce qu'ils pensent que je méprise les langues qu'on parle ici (à Boane)/ puisque tout le monde parle ces langues [...] alors quand ils voient une personne qui parle seulement le portugais/ ils pensent qu'elle méprise la langue du coin/ alors que ce n'est pas ça/ souvent les parents n'ont pas créé des conditions pour qu'on apprenne ces langues-là/ tel est mon cas. »

Dans l'esprit des voisins en question, le refus de parler portugais est un message pour contester l'inadéquation de cette langue par rapport à la situation informelle de communication. Pour eux, il paraît inconcevable de parler portugais entre voisins, dans une situation informelle, à la maison, 
et en plus à la campagne. À leurs yeux, il s'avère impensable qu'une personne du «coin » ne parle pas la langue du «coin». On peut donc parler d'une certaine revendication identitaire des langues locales au sein de la conscience linguistique du quartier.

Nous dirons donc avec Cécile Canut (1996 : 25) «qu'un usage n'existe pas sans sa représentation et que l'interaction entre les pratiques et la représentation de ces pratiques constitue un ensemble indissociable ».

Par ailleurs, nous avons constaté que les parents jouent un rôle important dans la rupture ou la continuité de la pratique des langues bantoues. En effet, certains parents, soucieux d'atteindre un statut social prestigieux, interdisent fortement (menaces, coups, répression violente, punition) à leurs enfants toute pratique de langue bantoue (même si souvent, c'est leur propre langue maternelle) et se contentent d'imposer le portugais, véritable politique linguistique familiale : «Quand nous étions petits/ nos parents nous ont toujours obligé à parler portugais/ alors nous avons grandi en parlant portugais/ du "ronga" on a eu quelques "miettes" avec des copains en dehors de la maison/ et quand nous allions rendre visite à nos grands-parents » (enquêté $\ll \mathrm{G} »)$.

Reconnaissons-le, « le plurilinguisme environnant est à la base des stratégies politiques des chefs de famille qui gèrent le capital linguistique familial, c'est-à-dire l'ensemble des langues possédées et utilisées ou non par les membres de la famille. Ils se sentent autant responsables, sinon plus, de l'avenir linguistique de leurs enfants que de leur éducation ou de leur avenir socio-économique, car la langue entre dans l'ensemble des éléments traditionnels à transmettre, au même titre que les valeurs morales, comme le respect du père, du grand frère, de la hiérarchie, des personnes âgées, etc. » (ibid. : 294).

Signalons que cet article ne représente qu'une étape de nos recherches qui sont toujours en cours. À travers cette étude de terrain, nous avons voulu donner un aperçu de la complexité de la situation linguistique au Mozambique et, en particulier, de la problématique du contact des langues, en ayant comme perspective la mise en relief des attitudes, des représentations, du positionnement des sujets qui se trouvent au «carrefour» de plusieurs langues. Nous invitons le lecteur familiarisé ou non avec le thème abordé, à dialoguer avec nous, à manifester ses idées, à apporter sa contribution de façon ouverte.

Université pédagogique de Maputo, Mozambique.

Université Eduardo Mondlane de Maputo, Mozambique. 


\section{BIBLIOGRAPHIE}

BORELL, J.

1990 Essai sur les représentations, Paris, Aubier.

BOYER, H.

1989 «Petit écran et représentations collectives », Le français dans le Monde, $222: 104-116$.

CAHEN, M.

1993a «Le Mozambique est-il un État-nation?», Institut d'études politiques de Bordeaux, Éditions du CNRS : 80-124.

1993b «Mozambique, Histoire, Géopolitique d'un pays sans nation », Institut d'études politiques de Bordeaux, Éditions du CNRS : 20-260.

Calvet, L. J.

1974 Linguistique et colonialisme, petit traité de glottophagie, Paris, Payot.

1987 La guerre des langues, Paris, Payot.

CANUT, C.

1996 Dynamiques linguistiques au Mali, Paris, Didier Érudition.

2000 «Subjectivité, imaginaires et fantasmes des langues : la mise en discours épilinguistique », Langage et Société, 93 : 71-97.

2001 «Créoles et dialectes, la typologie des variétés face aux dires des locuteurs », Traverses, $2: 387-410$.

Canut, C., ed.

1998 Imaginaires linguistiques en Afrique, Paris, L'Harmattan.

Erhrlich, M.-F., Tardieu, H. \& Cavazza, M.

1993 Les modèles mentaux, Paris, Masson.

Gonçalves, P.

1993 «Parler (du) portugais au Mozambique», La revue du livre, 113: 13-15.

Honwana, L. B.

1983 Contribuição para a definição de uma política linguística na R. P. M, Maputo, Secretaria de Estado da cultura.

JOUANEAU, D.

1995 Le Mozambique, Éditions Karthala.

Kathupa, J. M.

1985 «O panorama linguístico de Moçambique e a contribuição da linguística apropriada », Actas do l'encontro da Associação de linguistas, 1 : 317-328. 
Lemos, V. DE

1993 «Un mythe à Paris », La revue du Livre, 113 : 43-45.

LOPES, L.

1997 «Política linguística, princínpios e problemas », livraria universitária, UEM : $15-41$.

Martinho, M.

1995 A língua portuguesa em África : educação, ensino, formação, Evora, Pendor éditorial.

NoGueIRA, R. DE

1954 Temas de linguistica Bantou: não virão de um mesmo tronco as linguas bantas $e$ as indo-europeias? Lisboa, Instituto de linguas africanas e orientais.

Stroud, C. \& Gonçalves, P.

1998 «Panorama do português oral do Maputo», cadernos de pesquisa, 24 : 13-263.

Stroud, C. \& Tuzine, A.

1998 «Uso de línguas africanas no ensino: problemas e perspectivas, cadernos de pesquisa », UNDE, $26: 250-267$.

VAn Eys, T. \& SEqueira, E.

1997 «Desenvolvimento e mudar para melhor a partir do nivel em que estamos », Boletim informativo coordenação para a mulher no desenvolvimento, Forum da Mulher, $9: 26$.

\section{RÉSUMÉ}

Cet article s'inscrit dans notre projet de recherche sociolinguistique autour de la problématique des représentations des langues et des positionnements épilinguistiques des sujets, dans le cas spécifique du Mozambique. Si les études, allant dans ce sens, ne sont pas nombreuses en général, elles sont quasi inexistantes au Mozambique. Notre article s'articule en trois parties. Premièrement, nous proposons un témoignage relatif à l'étude des langues mozambicaines, où le linguiste est confronté à la difficulté de la tâche descriptive. Deuxièmement, nous présentons la situation linguistique du Mozambique et la manière dont elle se reflète dans la dynamique $d^{\prime}$ 'usages. Troisièmement, nous entendons illustrer la relation entre les pratiques linguistiques et leurs représentations, par l'analyse d'une " micro-situation » de langues en contact, celle d'une bourgade mi-urbaine et mi-rurale, à $30 \mathrm{~km}$ de la capitale. L'objectif est de déceler les attitudes des sujets, à travers leurs discours, sur les langues, sur eux-mêmes et sur leur rapport à ces langues. De cette approche de terrain, une question de fond émerge : I'environnement plurilingue, entraîne-t-il forcément une guerre des langues dans l'imaginaire linguistique des usagers? 


\section{ABSTRACT}

Language Contacts in the Sociolinguistic Context of Mozambique. - How are languages seen in Mozambique and how do speakers position themselves epilinguistically? Few in number in general, studies of this sort practically do not exist for Mozambique. An account is made of language studies in Mozambique, where linguists have difficulties describing the situation. The country,s linguistic situation is then presented as well as the way it reflects changing uses of languages. The relation between linguistic practices and ideas about them are illustrated by analyzing a "micro situation" of language contact in a semiurban, semirural town $30 \mathrm{~km}$. from the capital. The aim is to detect speakers, attitudes through what they say about languages, themselves and their relation to languages. A question emerges from this field approach: does the multilingual establishment necessarily lead to a language war in the imagination of language-users?

Mots-clés/Keywords: Mozambique, attitudes, diglossie, langues, plurilinguisme, représentations/Mozambique, attitudes, diglossia, languages, multilingualism. 